\title{
Synthesis of Zinc Oxide Nanostructures on Graphene/Glass Substrate via Electrochemical Deposition: Effects of Potassium Chloride and Hexamethylenetetramine as Supporting Reagents
}

\author{
Nur Ashikyn Hambali ${ }^{1}$ Abdul Manaf Hashim ${ }^{1}$
}

Received: 25 April 2015/ Accepted: 4 June 2015/Published online: 20 June 2015

(C) The Author(s) 2015. This article is published with open access at Springerlink.com

\begin{abstract}
The effects of the supporting reagents hexamethylenetetramine (HMTA) and potassium chloride (KCl) mixed in zinc nitrate hexahydrate $\left(\mathrm{Zn}\left(\mathrm{NO}_{3}\right)_{2} \cdot 6 \mathrm{H}_{2} \mathrm{O}\right)$ on the morphological, structural, and optical properties of the resulting $\mathrm{ZnO}$ nanostructures electrodeposited on graphene/glass substrates were investigated. The supporting reagent HMTA does not increase the density of nanorods, but it does remarkably improve the smoothness of the top edge surfaces and the hexagonal shape of the nanorods even at a low temperature of $75^{\circ} \mathrm{C}$. Hydroxyl $\left(\mathrm{OH}^{-}\right)$ions from the HMTA suppress the sidewall growth of non-polar planes and promote the growth of $\mathrm{ZnO}$ on the polar plane to produce vertically aligned nanorods along the $c$ axis. By contrast, the highly electronegative chlorine $\left(\mathrm{Cl}^{-}\right)$ions from the supporting reagent $\mathrm{KCl}$ suppress the growth of $\mathrm{ZnO}$ on the polar plane and promote the growth on non-polar planes to produce vertical stacking nanowall structures. HMTA was found to be able to significantly improve the crystallinity of the grown $\mathrm{ZnO}$ structures, as indicated by the observation of much lower FWHM values and a higher intensity ratio of the emission in the UV region to the emission in the visible region. Equimolar mixtures of $\mathrm{Zn}\left(\mathrm{NO}_{3}\right)_{2} \cdot 6 \mathrm{H}_{2} \mathrm{O}$ and the supporting reagents $\mathrm{HMTA}$ and $\mathrm{KCl}$ seem to provide the optimum ratio of concentrations for the growth of high-density, uniform $\mathrm{ZnO}$ nanostructures. The corresponding transmittances for such molar ranges are approximately 55-58 \% (HMTA) and 63-70 \% (KCl), which are acceptable for solar cell and optoelectronic devices.
\end{abstract}

Keywords Zinc oxide $\cdot$ Electrochemical deposition $\cdot$ Graphene $\cdot$ Nanorod $\cdot$ Nanowall

\section{Introduction}

The growth of highly crystalline metal oxides, such as zinc oxide $(\mathrm{ZnO})$, on arbitrary substrates such as glass, metal, plastic and other conventional insulating materials, such as silicon dioxide and silicon nitride, is very difficult, primarily because of the large lattice mismatch [1, 2]. Because the structures of these arbitrary substrates and conventional insulating materials are amorphous or polycrystalline, $\mathrm{ZnO}$ structures that are grown on these

Abdul Manaf Hashim

abdmanaf@utm.my

1 Malaysia-Japan International Institute of Technology, Universiti Teknologi Malaysia, Jalan Sultan Yahya Petra, 54100 Kuala Lumpur, Malaysia materials also typically tend to be amorphous or polycrystalline. Graphene is a two-dimensional hexagonal network of carbon atoms that is formed through the establishment of strong triangular $\sigma$ bonds of $s p^{2}$-hybridized orbitals $[3,4]$. This bonding structure is similar to that of the $c$ plane of a hexagonal crystalline structure and the (111) plane of the zincblende structure [5]. Therefore, the growth of $\mathrm{ZnO}$ on non-crystalline substrates using graphene as a template layer is feasible [6-9]. $\mathrm{ZnO}$ nanostructures and thin films on graphene are particularly interesting because these structures can imbue the graphene with additional functionality for the realization of advanced electronic and optoelectronic applications in photovoltaics, field emission devices, sensors, efficient energy conversion and storage devices, among others [10-14]. Graphene also offers considerable potential for novel electronic devices because of its extraordinary electrical, thermal, and 
mechanical properties, including a carrier mobility exceeding $10^{4} \mathrm{~cm}^{2} \mathrm{Vs}^{-1}$ and a thermal conductivity of $10^{3} \mathrm{~W} \mathrm{mK}^{-1}$ [15-19]. Therefore, by virtue of the excellent properties of graphene, the growth of $\mathrm{ZnO}$ nanostructures and thin films on graphene layers would enable the novel physical properties of both materials to be exploited in a wide variety of sophisticated device applications with flexible, wearable, and transferable capabilities $[10,20]$.

The most common methods of growing $\mathrm{ZnO}$ on graphene are vapor-phase techniques such as thermal evaporation $[8,9]$ and metal-organic vapor phase epitaxy (MOVPE) [21]. Vapor-phase methods are likely to involve high-temperature processes and are also considered to be high in cost. Additionally, because the growth process requires oxygen $\left(\mathrm{O}_{2}\right)$, especially for the growth of $\mathrm{ZnO}$ via thermal evaporation, the possibility that the graphene will be oxidized or etched out during growth is high because graphene oxidation can occur at temperatures as low as $450{ }^{\circ} \mathrm{C}$ [22]. Liquid-phase methods, such as electrochemical or hydrothermal deposition, appear promising for the low-temperature growth of $\mathrm{ZnO}$ on graphene with well-controlled growth rates and structural dimensions $[6,7,23,24]$. Although the growth of $\mathrm{ZnO}$ nanorods on graphene via a hydrothermal process has been reported, the need to use either seeded graphene or a pressurized container to induce growth is a significant disadvantage of this process. As an alternative, electrochemical deposition offers a feasible means of growing $\mathrm{ZnO}$ with high crystallinity and uniformity on a seedless graphene substrate with a simple process setup $[6,7,24]$. Recently, we have been examining the growth of $\mathrm{ZnO}$ nanorods on graphene on glass [25] because such a hybrid structure is expected to be promising for solar cell technology, which is a leading candidate for addressing the global need for inexpensive alternative energy sources.

Previously, we have reported the electrochemical growth of $\mathrm{ZnO}$ nanorods on graphene on glass using zinc nitrate hexahydrate as an electrolyte without any supporting reagents [25]. In that study, the effects of current density and temperature on the morphological, structural, and optical properties of the grown $\mathrm{ZnO}$ structures were investigated. The highest density of vertically aligned nanorods with few structural defects was obtained at a temperature and a current density of $75^{\circ} \mathrm{C}$ and $-0.1 \mathrm{~mA} \mathrm{~cm}{ }^{-2}$, respectively. However, higher temperatures of above $80{ }^{\circ} \mathrm{C}$ appear to be required for the generation of nanorods with a well-defined hexagonal shape and a smooth top edge surface. In this work, the effects of supporting reagents, i.e., hexamethylenetetramine (HMTA) and potassium chloride $(\mathrm{KCl})$, on the morphological, structural, and optical properties of the grown $\mathrm{ZnO}$ structures were systematically studied. The purpose of introducing these supporting reagents is not only to increase the conductivity of the solution used in the electrodeposition of the $\mathrm{ZnO}$ but also to enable the control of the resulting structures' morphology. It is well known that because of the positions of the $\mathrm{Zn}$ and $\mathrm{O}$ ions in the $\mathrm{ZnO}$ unit cell and the asymmetry of the hexagonal lattice around the unit cell center, the wurtzite phase of $\mathrm{ZnO}$ exhibits a finite dipole moment along the hexagonal $c$ axis. Because of this dipole moment, the $\mathrm{ZnO}$ (0001) surface becomes a "polar surface" and should be unstable. However, $\mathrm{ZnO}$ (0001) surfaces are commonly observed in $\mathrm{ZnO}$ nanostructures and thin films. Because of the presence of the dipole moment, the surface energy of the $\mathrm{ZnO}$ (0001) plane is higher than those of all low-

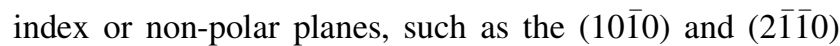
planes of the wurtzite $\mathrm{ZnO}$ crystal. The hydroxyl $\left(\mathrm{OH}^{-}\right)$and chlorine $\left(\mathrm{Cl}^{-}\right)$ions that are produced in the decomposition of HMTA and $\mathrm{KCl}$, respectively, have different electronegativities; specifically, $\mathrm{OH}^{-}$is less electronegative than $\mathrm{Cl}^{-}$. Therefore, their effects, particularly on the attraction/adsorption to the polar and non-polar planes of the $\mathrm{ZnO}$ crystal, should have a significant impact on the resulting morphological structures. Specifically, adsorption onto the side surfaces or non-polar surfaces of the $\mathrm{ZnO}$ will enhance the growth in the vertical direction, whereas capping on the basal plane or polar surfaces of the $\mathrm{ZnO}$ will promote the growth in the lateral direction.

\section{Experimental Procedures}

A monolayer of graphene on glass (Graphene Laboratory Inc., USA) was used as a substrate for the growth of $\mathrm{ZnO}$ nanostructures. The electrochemical deposition process was performed via cathodic electrochemical deposition using two electrodes, with a platinum $(\mathrm{Pt})$ wire acting as the anode and the monolayer graphene acting as the cathode [25]. A mixture of zinc nitrate hexahydrate $\left(\mathrm{Zn}\left(\mathrm{NO}_{3}\right)_{2} \cdot 6 \mathrm{H}_{2} \mathrm{O}\right)$ (Sigma-Aldrich, $\geq 99.0 \%$ purity) solution and a supporting reagent, i.e., either HMTA $\left(\mathrm{C}_{6} \mathrm{H}_{12} \mathrm{~N}_{4}\right)$ or $\mathrm{KCl}$, was used as the electrolyte. Both the anode and cathode were connected to an external direct current (DC) power supply. In this experiment, electrodeposition was performed under galvanostatic control, with the current density remaining fixed during the deposition. The growth was performed at a current density of $-0.1 \mathrm{~mA} \mathrm{~cm}^{-2}$ and a temperature of $75{ }^{\circ} \mathrm{C}$ because these conditions have been found to be optimal for obtaining the highest density of vertically aligned nanorods with few structural defects, as reported in Ref. [25]. The sample was inserted into the electrolyte at the beginning of the process, prior to the heating of the electrolyte from room temperature (RT) to $75{ }^{\circ} \mathrm{C}$. Growth was allowed to continue for $45 \mathrm{~min}$ after the electrolyte temperature reached $75{ }^{\circ} \mathrm{C}$. The grown structures were characterized using a field emission scanning electron microscopy (FESEM) apparatus (Hitachi SU8030) equipped with energy-dispersive X-ray (EDX) spectroscopy, X-ray diffraction (XRD, Bruker AXES 
D8 Advance), and photoluminescent (PL) spectroscopy (WiTec Alpha300R+) instruments and a UV-Vis spectrometer (Cary 5000).

\section{Results and Discussion}

Figure 1a-d shows top-view FESEM images of the $\mathrm{ZnO}$ structures grown on graphene using mixtures containing different concentrations of HMTA, whereas Fig. 1e-f shows similar images of the nanostructures obtained using mixtures containing different concentrations of $\mathrm{KCl}$. The molar ratio of $\mathrm{Zn}\left(\mathrm{NO}_{3}\right)_{2} \cdot 6 \mathrm{H}_{2} \mathrm{O}$ to the reagent, i.e., HMTA or $\mathrm{KCl}$, was set to $9: 1$ (denoted as 10\% HMTA or $\mathrm{KCl}$ ), 7:3 (denoted as $30 \%$ HMTA or $\mathrm{KCl}$ ), 1:1 (denoted as $50 \%$ HMTA or $\mathrm{KCl}$ ), and 1:9 (denoted as $90 \%$ HMTA or $\mathrm{KCl}$ ). Figure 1 shows that in general, an electrolyte mixed with HMTA tends to produce nanorods that are hexagonal in shape, whereas an electrolyte mixed with $\mathrm{KCl}$ tends to produce stacking nanowall structures. The densities of nanorods and nanowalls also appear to increase as the reagent concentration is increased from 10 to $50 \%$, as shown in Fig. 1a-c and Fig. 1e-g, respectively. However, the densities of the grown structures decrease once again when the reagent concentration in the electrolyte is further increased to a high value of $90 \%$, as shown in Fig. 1d, h. Furthermore, at a reagent concentration of $90 \%$, in the case of HMTA, the grown nanorod structures exhibit poorly defined hexagonal shapes with rough top edge surfaces, as shown in Fig. 1d, whereas in the case of $\mathrm{KCl}$, thin and sparsely distributed nanocluster-like structures are observed. The densities of the nanorods grown using electrolytes containing 10, 30, 50, and $90 \%$ HMTA were estimated to be approximately $2.90 \times 10^{8}, 4.15 \times 10^{8}$, $5.39 \times 10^{8}$, and $2.28 \times 10^{8} \mathrm{~cm}^{-2}$, respectively; these values are one order of magnitude lower than the nanorod density $\left(1.45 \times 10^{9} \mathrm{~cm}^{-2}\right)$ obtained using an electrolyte without HMTA at the same current density and temperature [25]. The nanorod density was determined by averaging the quantities of nanorods observed in three different regions on each sample, with a total area of $125 \mu \mathrm{m}^{2}$ for each region; the value thus obtained was then normalized to square centimeters $\left(\mathrm{cm}^{2}\right)$. To determine the numbers of nanorods in such large regions, the numbers of nanorods observed in five FESEM surface morphological images were summed, where each image had dimensions of approximately $5 \mu \mathrm{m} \times 5 \mu \mathrm{m}$. In an analysis of the EDX spectra, only zinc $(\mathrm{Zn})$, oxygen $(\mathrm{O})$, and carbon $(\mathrm{C})$ elements were detected in all of the grown samples, and the total compositional atomic percentages of $\mathrm{Zn}$ and $\mathrm{O}$ were estimated to be above $95 \%$.

The basic cathodic electrodeposition reactions of $\mathrm{ZnO}$ formation from a $\mathrm{Zn}\left(\mathrm{NO}_{3}\right)_{2} \cdot 6 \mathrm{H}_{2} \mathrm{O}$ solution can be expressed as follows $[6,7,25]$ :

Cathode:

$\mathrm{Zn}\left(\mathrm{NO}_{3}\right)_{2} \rightarrow \mathrm{Zn}^{2+}+2 \mathrm{NO}_{3}^{-}$

$\mathrm{NO}_{3}^{-}+\mathrm{H}_{2} \mathrm{O}+2 \mathrm{e}^{-} \rightarrow \mathrm{NO}_{2}^{-}+2 \mathrm{OH}^{-}$

$\mathrm{Zn}^{2+}+2 \mathrm{OH}^{-} \rightarrow \mathrm{Zn}(\mathrm{OH})_{2}$

$\mathrm{Zn}(\mathrm{OH})_{2} \rightarrow \mathrm{ZnO}+\mathrm{H}_{2} \mathrm{O}$

Anode:

$\mathrm{H}_{2} \mathrm{O} \rightarrow 1 / 2 \mathrm{O}_{2}+2 \mathrm{H}^{+}+2 \mathrm{e}^{-}$.

With reference to the above reactions, additional hydroxyl $\left(\mathrm{OH}^{-}\right)$ions are further obtained via the chemical reaction of HMTA with water, as shown below [6, 7].

$\mathrm{C}_{6} \mathrm{H}_{12} \mathrm{~N}_{4}+6 \mathrm{H}_{2} \mathrm{O} \rightarrow \mathrm{COH}_{2}+4 \mathrm{NH}_{3}$

$\mathrm{NH}_{3}+\mathrm{H}_{2} \mathrm{O} \rightarrow \mathrm{NH}_{4}^{+}+\mathrm{OH}^{-}$.
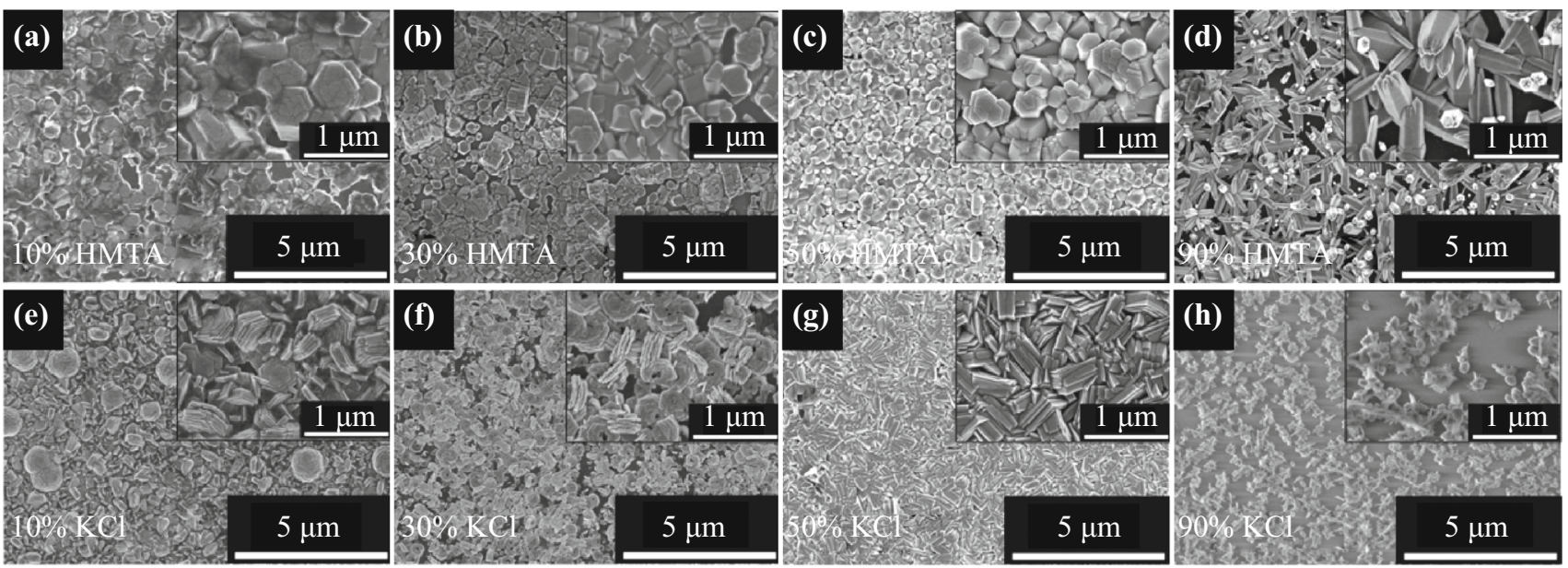

Fig. 1 Top-view FESEM images of the ZnO structures grown in a-d HMTA-containing and $\mathbf{e}-\mathbf{h}$ KCl-containing electrolytes 
The FESEM images shown in Fig. 1a-d suggest that HMTA percentages of approximately $50 \%$ and below are most favorable for allowing the HMTA to act as a mineralizer to supply additional $\mathrm{OH}^{-}$ions during the formation of the $\mathrm{ZnO}$ structures, thereby giving rise to nanorods with well-defined hexagonal shapes and smooth top edge surfaces $[6,7]$. Based on the presented results, $50 \%$ HMTA appears to be the optimal concentration, as it yields a high density of nanorods with good uniformity. Generally, it can be said that the adsorption of $\mathrm{OH}^{-}$ions onto the side surfaces or nonpolar surfaces of the $\mathrm{ZnO}$ enhances vertical $\mathrm{ZnO}$ growth, leading to nanorod formation. At low HMTA concentrations, it is speculated that the correspondingly small number of $\mathrm{OH}^{-}$ions is not only insufficient to coordinate and bridge an adequate number of $\mathrm{Zn}^{2+}$ ions to form a uniform $\mathrm{ZnO}$ structure but also insufficient to attach to the non-polar side facets to facilitate vertical growth in the [0001] direction. This hypothesis is supported by the FESEM images shown in Fig. 1a, b, in which fewer vertical nanorod structures are observed. As the concentration of HMTA increases to $50 \%$, it seems to create a balanced number of $\mathrm{OH}^{-}$and $\mathrm{Zn}^{2+}$ ions to perform the described activities, resulting in high-density, uniform nanorod structures, as shown in Fig. 1c. However, at high HMTA concentrations, the presence of excess $\mathrm{OH}^{-}$ ions compared with $\mathrm{Zn}^{2+}$ seems to create an unbalanced condition in which the excess $\mathrm{OH}^{-}$ions play a significant role in coordinating and bridging $\mathrm{Zn}^{2+}$ ions to form $\mathrm{ZnO}$ structures rather than attaching to the non-polar side facets to facilitate the vertical growth in the [0001] direction, thus resulting in the formation of fewer vertically aligned nanorods, as shown in Fig. 1d.

When the $\mathrm{KCl}$ reagent was added to the electrolyte, instead of nanorods, vertically aligned nanowall structures were obtained. As mentioned in the previous section, $\mathrm{ZnO}$ has two types of crystal planes, which are classified as polar

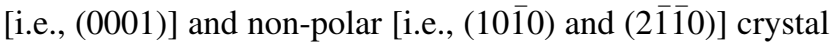
planes. The highly electronegative chlorine $\left(\mathrm{Cl}^{-}\right)$ions generated in the chemical reactions with $\mathrm{KCl}$ should be easily attracted to the polar (0001) $\mathrm{ZnO}$ crystal plane. Once a layer of $\mathrm{Cl}^{-}$ions has adsorbed and formed on the (0001) crystal plane, the subsequent deposition of $\mathrm{ZnO}$ can occur only on the non-polar crystal planes, which causes the individual $\mathrm{ZnO}$ hexagonal crystals to grow sideways, forming two-dimensional (2D) structures, i.e., stacking nanowall structures [26]. This expectation seems to indicate that $\mathrm{Cl}^{-}$ions could act as a capping agent on the (0001) plane and thus control the formation of 2D nanowall $\mathrm{ZnO}$ structures, promoting growth toward the non-polar planes [26, 27]. It is speculated that at low $\mathrm{KCl}$ concentrations the correspondingly small number of $\mathrm{Cl}^{-}$ions may be insufficient to attach to the polar side facets to facilitate lateral growth in the [1010] and [2 $\overline{1} \overline{1} 0]$ directions. This hypothesis is supported by the
FESEM images shown in Fig. 1e, f, in which fewer vertical nanowall structures and several vertical nanorods are observed. As the $\mathrm{KCl}$ concentration increases to $50 \%$, the grown structures become dominated by vertical nanowall structures with improved uniformity, as shown in Fig. 1g, suggesting that this is the optimal amount of $\mathrm{Cl}^{-}$for achieving capping activity on the polar surfaces. However, at a high $\mathrm{KCl}$ concentration, the existence of an excess number of $\mathrm{Cl}^{-}$ions compared with $\mathrm{Zn}^{2+}$ appears to create unbalanced conditions in which excess capping activity may inhibit $\mathrm{ZnO}$ nucleation, thereby resulting in the formation of thin and sparsely distributed $\mathrm{ZnO}$ nanoclusters, as shown in Fig. 1h. These results suggest that approximately $50 \%$ of $\mathrm{KCl}$ is most favorable to act as a capping agent to suppress the growth of $\mathrm{ZnO}$ in the polar plane, resulting in the growth of $\mathrm{ZnO}$ toward the non-polar planes.

Figure $2 \mathrm{a}, \mathrm{b}$ shows the XRD spectra of the $\mathrm{ZnO}$ structures electrodeposited in the HMTA- and $\mathrm{KCl}$-containing mixtures, respectively, with various concentrations. The XRD patterns show that the grown $\mathrm{ZnO}$ exhibits wurtzite structures, as indicated by the observation of the three main (100), (002), and (101) peaks of ZnO (JCPDS Card No. 36-1451). As shown in Fig. 2a, the intensity of the $\mathrm{ZnO}$ (002) peak is much higher than those of the other $\mathrm{ZnO}$ peaks, indicating the highly oriented growth of the $\mathrm{ZnO}$ along the $c$ axis, particularly for the nanorod structures grown in the HMTAcontaining mixtures. The formation of $c$-axis-oriented crystal structures can be attributed to the stabilization of the polar (0001) $\mathrm{ZnO}$ surface and the existence of additional $\mathrm{OH}^{-}$ions suppressing the sidewall growth, resulting in the enhancement of $\mathrm{ZnO}$ formation along the direction of the polar surface. As shown in Fig. 2b, for the $\mathrm{ZnO}$ structures that formed in the presence of $\mathrm{KCl}$, the intensity of the $\mathrm{ZnO}$ (002) peak is much weaker and the intensities of the other two peaks, i.e., (100) and (101), are relatively strong, especially for a $\mathrm{KCl}$ concentration of $50 \%$. This finding is attributed to the promotion of growth toward the non-polar direction, i.e., the formation of nanowall structures, as revealed by the FESEM images. However, no peaks can be detected for the samples grown at $90 \% \mathrm{KCl}$, most likely because of the low density and low crystallinity of the thin and sparsely distributed nanocluster structures that were grown under these conditions. Figure $2 \mathrm{c}$ compares the intensities of the $\mathrm{ZnO}$ (002) peaks for the samples grown in HMTA- and $\mathrm{KCl}$-containing mixtures with various concentrations. It is clearly observed that the intensities of the (002) peak for the samples grown using HMTA are much higher than those for the samples grown using $\mathrm{KCl}$. It is also evident that for both HMTA and $\mathrm{KCl}$ individually, the intensities exhibit no significant difference among the tested concentrations. Moreover, the intensities of the $\mathrm{ZnO}(002)$ peaks of structures grown with supporting reagents are much 

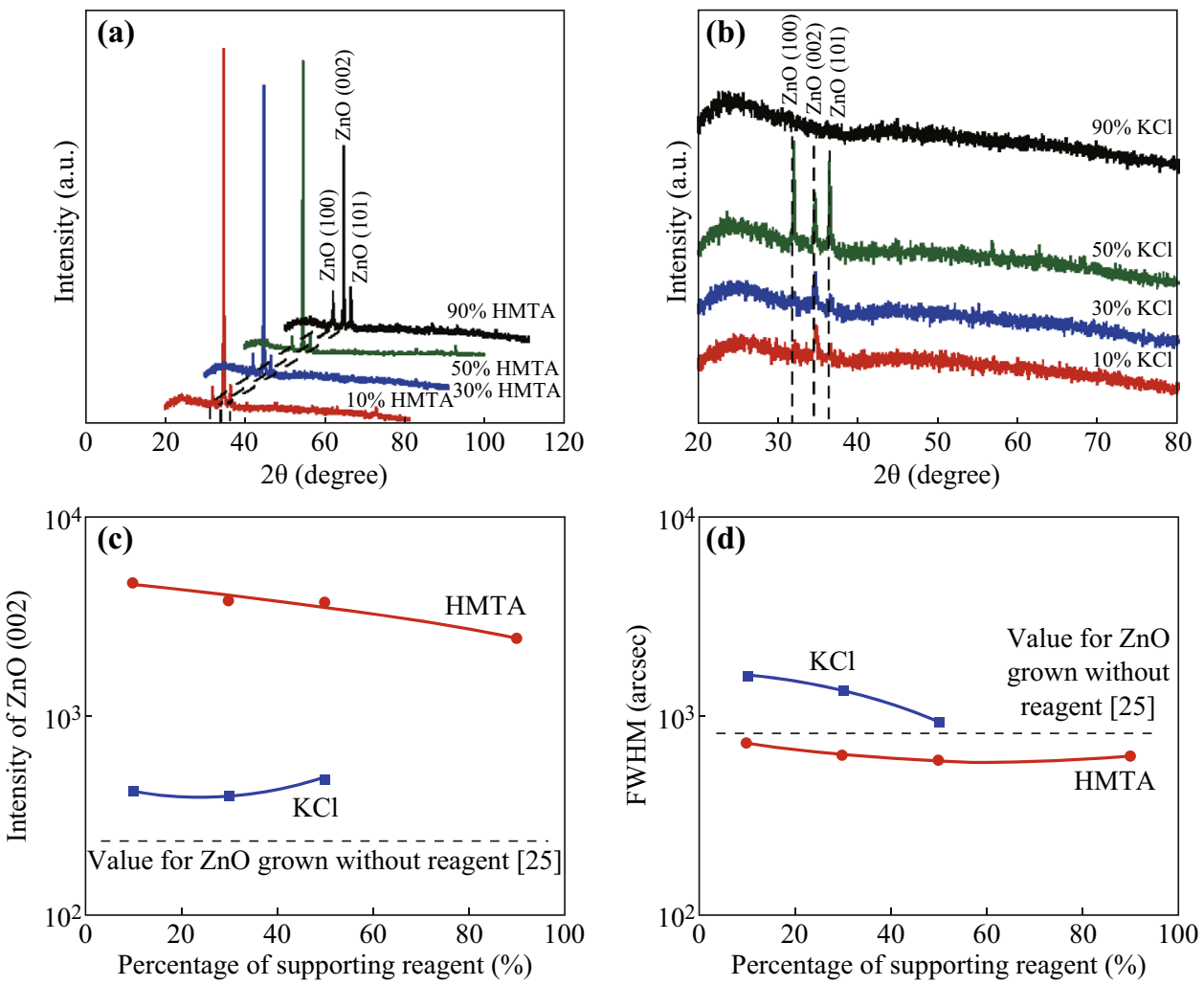

Fig. 2 XRD patterns of the $\mathrm{ZnO}$ structures grown in a HMTA-containing and $\mathbf{b} \mathrm{KCl}$-containing electrolytes. $\mathbf{c}$ Intensities and $\mathbf{d}$ FWHM values of the $\mathrm{ZnO}(002)$ peak

higher than those of structures grown without any supporting reagent, as presented in Ref. [25], for the same current density and temperature.

Figure 2d compares the FWHM values of the (002) peaks for the corresponding grown structures. All samples grown in HMTA-containing mixtures exhibit lower values compared with the corresponding sample grown in a mixture without a reagent [25]; however, this finding simply indicates the better crystallinity of the structures grown using HMTA as a supporting reagent for the same current density and temperature. Here, the following important conclusions can be drawn: (i) the supporting reagent HMTA does not increase the density of the grown nanorods, but it does improve the smoothness of the top edge surfaces of the nanorods, even at a low temperature of $75^{\circ} \mathrm{C}$; (ii) HMTA suppresses the sidewall growth, i.e., the growth along the non-polar planes, while also promoting the growth of $\mathrm{ZnO}$ on the polar plane to produce vertically aligned nanorods along the $c$ axis; (iii) by contrast, the supporting reagent $\mathrm{KCl}$ suppresses the growth of $\mathrm{ZnO}$ on the polar plane, i.e., along the $c$ axis, while promoting the growth on the non-polar planes to produce vertically stacking nanowall structures; (iv) HMTA tends to increase the thickness of the grown structures due to the enhancement of the growth along the $c$ axis, as indicated by the observation of a high-intensity (002) peak, whereas $\mathrm{KCl}$ cannot significantly increase the thickness because it enhances the growth toward the nonpolar planes; and (v) HMTA is able to improve the crystallinity of the grown $\mathrm{ZnO}$ structures, as indicated by the observation of lower FWHM values.

Figure $3 \mathrm{a}$, b shows the RT PL spectra of the $\mathrm{ZnO}$ nanostructures grown on graphene with the addition of HMTA and $\mathrm{KCl}$, respectively, as supporting reagents at various concentrations. Two distinct emission bands can be observed. The first band is located in the ultraviolet (UV) region, with peaks in the range of $376-378 \mathrm{~nm}$, and the other is located in the visible region, with peaks in the range of 541-571 nm. This UV emission is also known as near-band edge (NBE) emission and can be regarded as an intrinsic property of the wurtzite crystal structure of $\mathrm{ZnO}$, originating from exciton recombination [28]. The second emission band, in the visible region, has been reported to be related to the radial recombination of photon-generated holes with the single ionized charges of local defects such as $\mathrm{O}$ vacancies or $\mathrm{Zn}$ interstitials [29-32]. A stronger UV emission peak indicates good optical properties, which may be attributed to a lower defect concentration. Figure 3c summarizes the relative RT PL intensity ratio of the emission in the UV region to that in the visible region, denoted by $I_{\mathrm{UV}} / I_{\mathrm{VIS}}$. All structures grown with the addition of reagents exhibit much higher intensity ratios compared with the corresponding structures grown without the addition of a supporting reagent [25]; this is particularly 

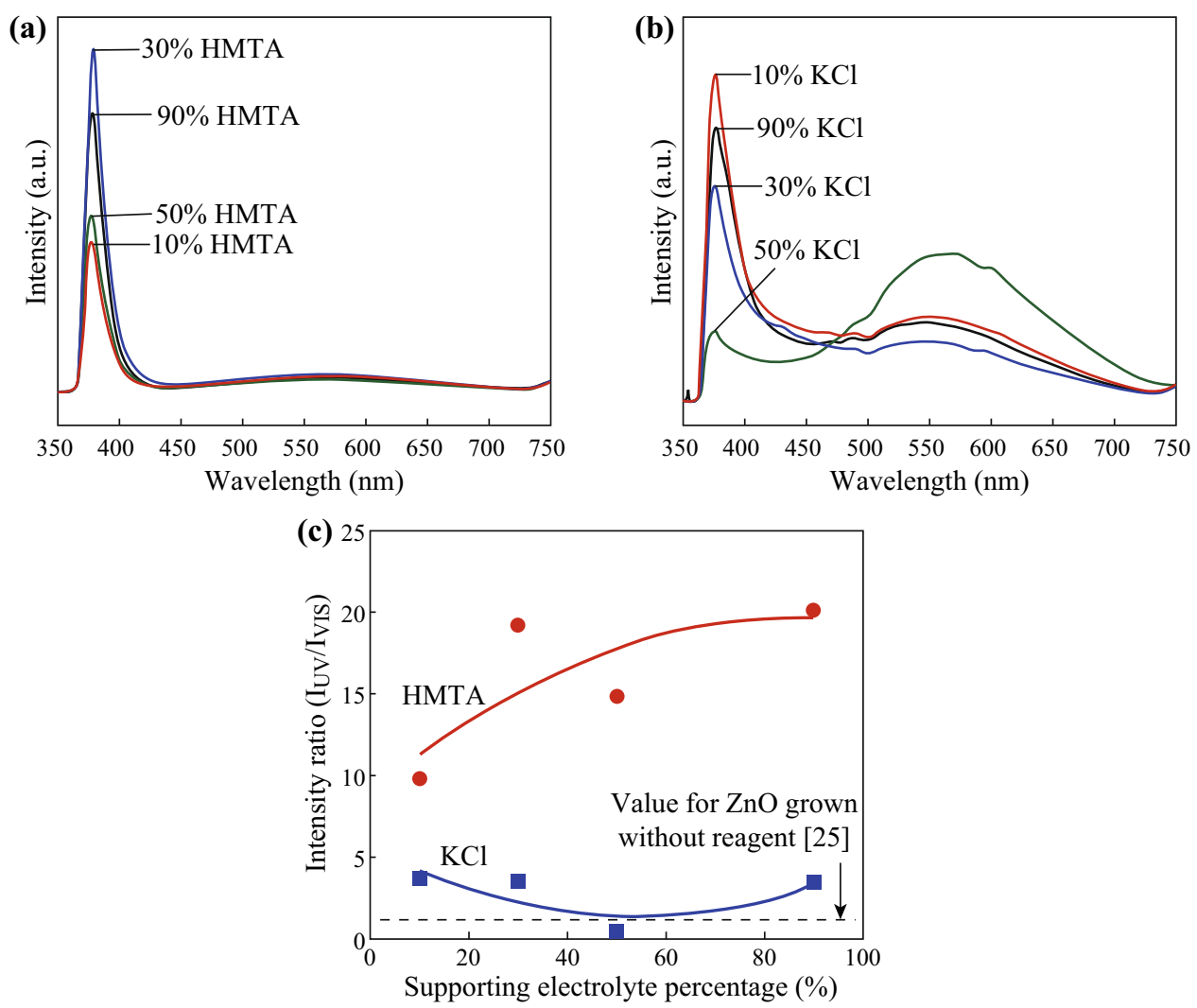

Fig. 3 PL spectra of the ZnO structures grown in a HMTA-containing and $\mathbf{b}$ KCl-containing electrolytes. $\mathbf{c}$ Intensity ratio of the UV emission to the visible emission, $I_{\mathrm{UV}} / I_{\mathrm{VIS}}$

pronounced for the samples grown using HMTA-containing mixtures. The samples grown with the addition of HMTA also exhibit higher values of the intensity ratio compared with the samples grown with the addition of $\mathrm{KCl}$. Thus, it can be concluded that the use of HMTA-containing mixtures for $\mathrm{ZnO}$ growth tends to result in fewer structural defects in the obtained $\mathrm{ZnO}$ nanostructures [33, 34].

Figure $4 a, b$ shows the optical transmittance spectra of the $\mathrm{ZnO}$ structures grown in mixtures containing various concentrations of HMTA and $\mathrm{KCl}$, respectively. The relationship between the supporting reagent concentration and the corresponding transmittance at a wavelength of $600 \mathrm{~nm}$ is shown in Fig. 4c. It was shown in the previous section that the optimal percentages of both reagents for the production of uniform $\mathrm{ZnO}$ structures are approximately $50 \%$ and below. As shown in Fig. 4c, the corresponding transmittances for these molar ranges are approximately 55-58 \% (HMTA) and 63-70\% $(\mathrm{KCl})$, which are acceptable for solar cell and optoelectronic devices. The transmittances of the $\mathrm{ZnO}$ structures grown with the addition of HMTA are lower than those of the structures grown without the addition of a reagent [25]; this is most likely attributable to the greater thicknesses of these structures. The structures grown with the addition of $\mathrm{KCl}$ exhibit higher transmittance values compared with the other structures, i.e., those grown with the addition of HMTA or without the addition of a reagent. This difference can presumably be attributed to the fact that although the density of the nanowall structures is high, these structures are also thinner. No significant difference is observed between the samples grown at the lowest (10\%) and highest (90\%) HMTA concentrations, indicating that HMTA addition does not strongly affect the thickness of the obtained $\mathrm{ZnO}$ nanostructures. The high value of transmittance observed for the sample grown with $90 \%$ $\mathrm{KCl}$ is most likely attributable to the low density and sparse distribution of the thin nanocluster structures.

Finally, it is worthwhile to briefly consider the other possible amines that can be explored for use as reagents to increase the density of vertically aligned nanorods. Polyethylenimine (PEI), a non-polar polymer with many amino side groups $\left(-\mathrm{NH}_{2}\right)$, has the ability to become protonated, thereby becoming positively charged, over a wide range of $\mathrm{pH}$ values (3-11). Therefore, adjusting the $\mathrm{pH}$ of the growth solution to fall into the range that leads to the protonation of PEI can result in highly positive PEI that adsorbs strongly onto the negatively charged surfaces of the $\mathrm{ZnO}$ due to electrostatic attraction. It is well known that the isoelectric point of $\mathrm{ZnO}$ occurs at a $\mathrm{pH}$ of approximately 9.5; hence, the sign of the $\mathrm{ZnO}$ surface sites is predominantly positive or predominantly negative for $\mathrm{pH}$ values below or above the isoelectric point, respectively. Thus, it may be possible to 

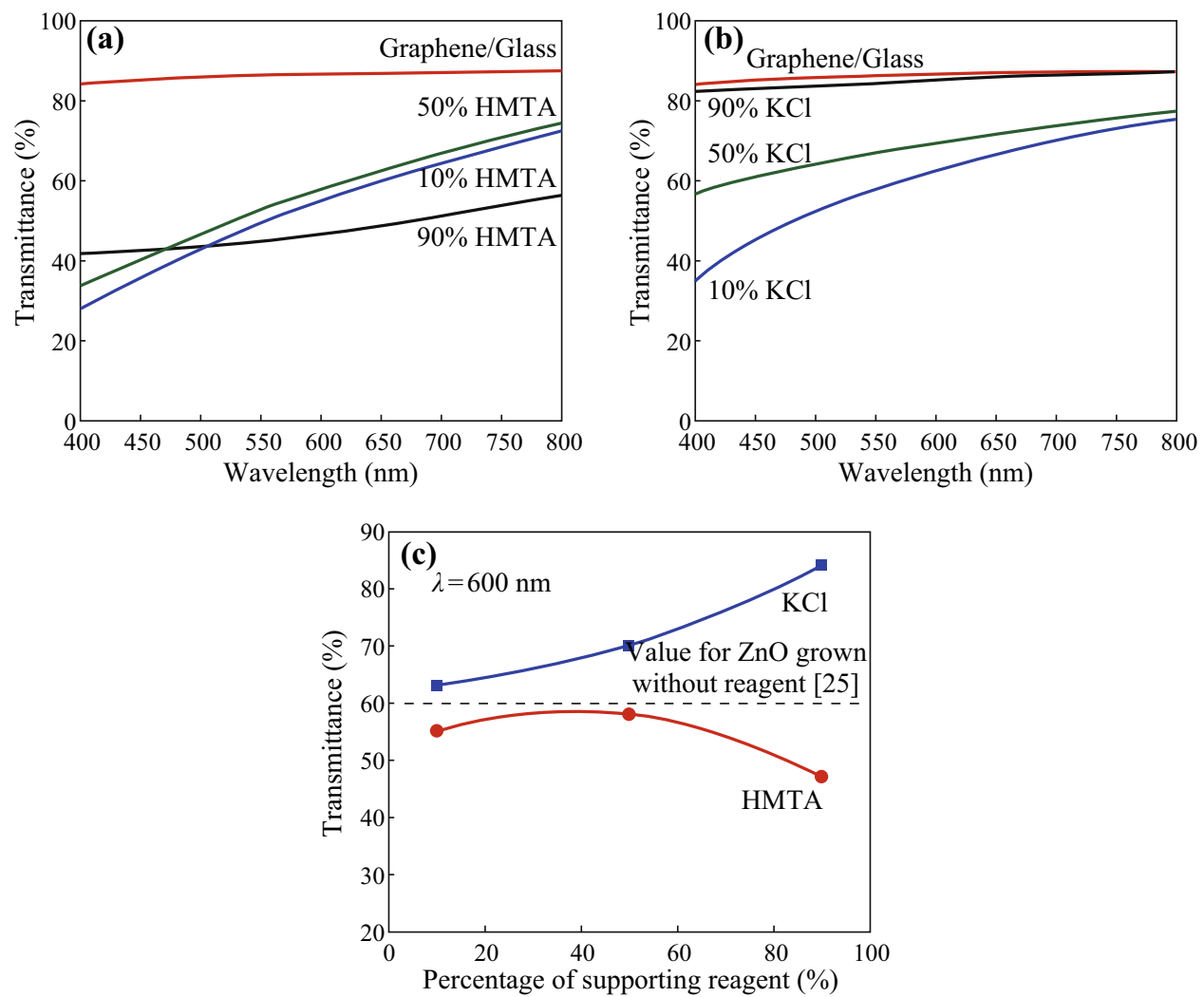

Fig. 4 Optical transmittances of the $\mathrm{ZnO}$ structures grown in a HMTA-containing and $\mathbf{b} \mathrm{KCl}$-containing electrolytes. $\mathbf{c}$ Transmittance at a wavelength of $600 \mathrm{~nm}$

enhance the vertical growth of high-density $\mathrm{ZnO}$ nanorods on graphene. Alternatively, citrate ions, which are characterized by three negative charges, are also good candidates for use as reagents to realize nanowall or nanoplate structures. Citrate ions may have the ability to strongly and specifically adsorb to the $\mathrm{Zn}^{2+}$ ions on the (0001) surface, thereby hindering the growth along the [0001] direction and forcing the growth to proceed along the $[10 \overline{1} 0]$ and $[2 \overline{1} \overline{1} 0]$ directions.

\section{Conclusions}

The effects of the supporting reagents HMTA and $\mathrm{KCl}$ on the morphological, structural, and optical properties of $\mathrm{ZnO}$ structures grown on monolayer graphene substrates were systematically investigated. It was observed that the two reagents produce different morphological structures because of the different roles played by the $\mathrm{OH}^{-}$ions from HMTA and the $\mathrm{Cl}^{-}$ions from $\mathrm{KCl}$. Equimolar concentrations of $\mathrm{Zn}\left(\mathrm{NO}_{3}\right)_{2} \cdot 6 \mathrm{H}_{2} \mathrm{O}$ and the supporting reagents appear to be optimal for the growth of high-density $\mathrm{ZnO}$ nanorods and nanowalls. Moreover, adding a supporting reagent, especially HMTA, to the electrolyte for the growth of $\mathrm{ZnO}$ appears to improve the morphological and crystalline properties of the grown nanorods. The measured transmittances were approximately 55-58 \% (HMTA) and 63-70\% (KCl), which are acceptable values for solar cell and optoelectronic devices. The obtained $\mathrm{ZnO}$ nanorods and nanowalls are also suitable to serve as low lattice mismatch seeds for the growth of gallium nitride $(\mathrm{GaN})$ for the preparation of II-VI and III-V materials on insulators.

Acknowledgments NAH thanks the Malaysia-Japan International Institute of Technology for providing a scholarship. This work was funded by Nippon Sheet Glass Corp.; the Hitachi Foundation; the Malaysia-Japan International Institute of Technology; Universiti Teknologi Malaysia; the Malaysian Ministry of Science, Technology and Innovation; and the Malaysian Ministry of Education through various research Grants.

Open Access This article is distributed under the terms of the Creative Commons Attribution 4.0 International License (http://creativecommons.org/licenses/by/4.0/), which permits unrestricted use, distribution, and reproduction in any medium, provided you give appropriate credit to the original author(s) and the source, provide a link to the Creative Commons license, and indicate if changes were made.

\section{References}

1. M. Wilander, O. Nur, N. Bano, K. Sultana, Zinc oxide nanorodbased heterostructures on solid and soft substrates for white-lightemitting diode applications. New J. Phys. 11, 125020 (2009). doi:10.1088/1367-2630/11/12/125020 
2. B.H. Kim, J.W. Kwon, Metal-catalyst for low-temperature growth of controlled zinc oxide nanowires on arbitrary substrates. Sci. Rep-UK 4, 4379 (2014). doi:10.1038/srep04379

3. A.K. Geim, K.S. Novoselov, The rise of graphene. Nat. Mater. 6, 183-191 (2007). doi:10.1038/nmat1849

4. A.K. Geim, Graphene: status and prospects. Science 324, 1530-1534 (2009). doi:10.1126/science.1158877

5. B. Astuti, M. Tanikawa, S.F.A. Rahman, K. Yasui, A.M. Hashim, Graphene as a buffer layer for silicon carbide-on-insulator structures. Materials 5(11), 2270-2279 (2012). doi:10.3390/ma5112270

6. N.S.A. Aziz, T. Nishiyama, N.I. Rusli, M.R. Mahmood, K. Yasui, A.M. Hashim, Seedless growth of zinc oxide flower-shaped structures on multilayer graphene by electrochemical deposition. Nanoscale Res. Lett. 9, 337 (2014). doi:10.1186/1556-276X-9-337

7. N.S.A. Aziz, M.R. Mahmood, K. Yasui, A.M. Hashim, Seed/catalyst-free vertical growth of high-density electrodeposited zinc oxide nanostructures on a single-layer graphene. Nanoscale Res. Lett. 9, 95 (2014). doi:10.1186/1556-276X-9-95

8. N.F. Ahmad, N.I. Rusli, M.R. Mahmood, K. Yasui, A.M. Hashim, Seed/catalyst-free growth of zinc oxide nanostructures on multilayer graphene by thermal evaporation. Nanoscale Res. Lett. 9, 83 (2014). doi:10.1186/1556-276X-9-83

9. N.F. Ahmad, K. Yasui, A.M. Hashim, Seed/catalyst-free growth of zinc oxide on graphene by thermal evaporation: effects of substrate inclination angles and graphene thicknesses. Nanoscale Res. Lett. 10, 10 (2015). doi:10.1186/s11671-014-0716-Z

10. K.S. Novoselov, A.K. Geim, S.V. Morozov, D. Jiang, M.I. Katsnelson, I.V. Grigorieva, S.V. Dubonos, A.A. Firsov, Two-dimensional gas of massless Dirac fermions in graphene. Nature 438, 197-200 (2005). doi:10.1038/nature04233

11. B. Kumar, K.Y. Lee, H.-K. Park, S.J. Chae, Y.H. Lee, S.-W. Kim, Controlled growth of semiconducting nanowire, nanowall, and hybrid nanostructures on graphene for piezoelectric nanogenerators. ACS Nano 5(5), 4197-4204 (2011). doi:10.1021/nn200942s

12. C.J. Lee, T.J. Lee, S.C. Lyu, Y. Zhang, H. Ruh, H.J. Lee, Field emission from well-aligned zinc oxide nanowires grown at low temperature. Appl. Phys. Lett. 81(19), 3648 (2002). doi:10.1063/ 1.1518810

13. D. Choi, M.-Y. Choi, W.-M. Choi, H.-J. Shin, H.-K. Park, J.-S. Seo, J. Park, S.-M. Yoon, S.J. Chae, Y.H. Lee, Fully rollable transparent nanogenerators based on graphene electrodes. Adv. Mater. 22(19), 2187-2192 (2010). doi:10.1002/adma.200903815

14. J.O. Hwang, D.H. Lee, J.Y. Kim, T.H. Han, B.H. Kim, M. Park, K. No, S.O. Kim, Vertical $\mathrm{ZnO}$ nanowires/graphene hybrids for transparent and flexible field emission. J. Mater. Chem. 21(10), 3432 (2011). doi:10.1039/C0JM01495H

15. K.S. Novoselov, V.I. Falko, L. Colombo, P.R. Gellert, M.G. Schwab, K. Kim, A roadmap for graphene. Nature 490, 192-200 (2012). doi:10.1038/nature11458

16. Y. Zhang, Y.-W, Tan, H.L. Stormer, P. Kim, Experimental observation of the quantum Hall effect and Berry's phase in graphene. Nature 438, 201-204 (2005). doi:10.1038/nature04235

17. K.S. Kim, Y. Zhao, H. Jang, S.Y. Lee, J.M. Kim, K.S. Kim, J.-H. Ahn, P. Kim, J.-Y. Choi, B.H. Hong, Large-scale pattern growth of graphene films for stretchable transparent electrodes. Nature 457, 706-710 (2009). doi:10.1038/nature07719

18. A.A. Balandin, S. Ghosh, W. Bao, I. Calizo, D. Teweldebrhan, F. Miao, C.N. Lau, Superior thermal conductivity of single-layer graphene. Nano Lett. 8, 902-907 (2008). doi:10.1021/n10731872

19. S.F.A. Rahman, S. Kasai, A.M. Hashim, Room temperature nonlinear operation of a graphene-based three-branch nanojunction device chemical doping. Appl. Phys. Lett. 100, 193116 (2012). doi:10.1063/1.4711035
20. K. Chung, C.H. Lee, G.C. Yi, Transferable GaN layers grown on $\mathrm{ZnO}$-coated graphene for optoelectronic devices. Science 330, 655-657 (2010). doi:10.1126/science.1195403

21. Y.-J. Kim, J.-H. Lee, G.C. Yi, Vertically aligned $\mathrm{ZnO}$ nanostructures grown on graphene layers. Appl. Phys. Lett. 95(21), 213101 (2009). doi:10.1063/1.3266836

22. L. Liu, S. Ryu, M.R. Tomasik, E. Stolyarova, N. Jung, M.S. Hybertsen, M.L. Steigerwald, L.E. Brus, G.W. Flynn, Graphene oxidation: thickness-dependent etching and strong chemical doping. Nano Lett. 8, 1965-1970 (2008). doi:10.1021/n10808684

23. Y.-J. Kim, A. Yoon, M. Kim, G.-C. Yi, C. Liu, Hydrothermally grown $\mathrm{ZnO}$ nanostructures on few-layer graphene sheets. Nanotechnology 22(24), 245603 (2011). doi:10.1088/0957-4484/22/ $24 / 245603$

24. C. Xu, B.-S. Kim, J.-H. Lee, M. Kim, S.W. Hwang, B.L. Choi, E.K. Lee, J.M. Kim, D. Whang, Seed-free electrochemical growth of $\mathrm{ZnO}$ nanotube arrays on single-layer graphene. Mater. Lett. 72, 25-28 (2012). doi:10.1016/j.matlet.2011.12.057

25. N.A. Hambali, H. Yahaya, M.R. Mahmood, T. Terasako, A.M. Hashim, Synthesis of zinc oxide nanostructures on graphene/glass substrate by electrochemical deposition: effects of current density and temperature. Nanoscale Res. Lett. 9, 609 (2014). doi:10. 1186/1556-276X-9-609

26. D. Pradhan, S. Sindhwani, Parametric study on dimensional control of $\mathrm{ZnO}$ nanowalls and nanowires by electrochemical deposition. Nanoscale Res. Lett. 5, 1727-1736 (2010). doi:10. 1007/s11671-010-9702-2

27. H.Y. Yang, Y.S. No, J.Y. Kim, T.W. Kim, Effect of potassium chloride concentration on the structural and optical properties of $\mathrm{ZnO}$ nanorods grown on glass substrates coated with indium tin oxide film. Jpn. J. Appl. Phys. 51(6), 06FG13 (2012). doi:10. 1143/JJAP.51.06FG13

28. K. Mahmood, S.S. Park, H.J. Sung, Enhanced photoluminescence, Raman spectra and field-emission behavior of indiumdoped $\mathrm{ZnO}$ nanostructures. J. Mater. Chem. C 1, 3138-3149 (2013). doi:10.1039/c3tc00082f

29. J.-J. Wu, S.-C. Liu, Low-temperature growth of well-aligned $\mathrm{ZnO}$ nanorods by chemical vapor deposition. Adv. Mater. 14(3), 215-218 (2002). doi:10.1002/1521-4095(20020205)14:3<215: AID-ADMA215>3.0.CO;2-J

30. A. Umar, S.H. Kim, J.H. Kim, A. Al-Hajry, Y.B. Hahn, Temperature-dependant non-catalytic growth of ultraviolet-emitting $\mathrm{ZnO}$ nanostructures on silicon substrate by thermal evaporation process. J. Alloys Compd. 463, 516-521 (2008). doi:10.1016/j. jallcom.2007.09.065

31. K.M.K. Srivatsa, D. Chikara, M.S. Kumar, Synthesis of aligned $\mathrm{ZnO}$ nanorod array on silicon and sapphire substrates by thermal evaporation technique. J. Mater. Sci. Technol. 27(8), 701-706 (2011). doi:10.1016/S1005-0302(11)60129-1

32. N.K. Hassan, M.R. Hashim, Y. Al-Douri, K. Al-Heuseen, Current dependence growth of $\mathrm{ZnO}$ nanostructures by electrochemical deposition technique. Int. J. Electrochem. Sci. 7(5), 4625-4635 (2012)

33. M. Ghosh, A.K. Raychaudhuri, Shape transition in $\mathrm{ZnO}$ nanostructures and its effect on blue-green photoluminescence. Nanotechnology 19(44), 1-7 (2008). doi:10.1088/0957-4484/19/44/ 445704

34. X.L. Xu, S.P. Lau, J.S. Chen, G.Y. Chen, B.K. Tay, Polycrystalline $\mathrm{ZnO}$ thin films on $\mathrm{Si}(100)$ deposited by filtered cathodic vacuum arc. J. Cryst. Growth 223(1-2), 201-205 (2001). doi:10. 1016/S0022-0248(01)00611-X 